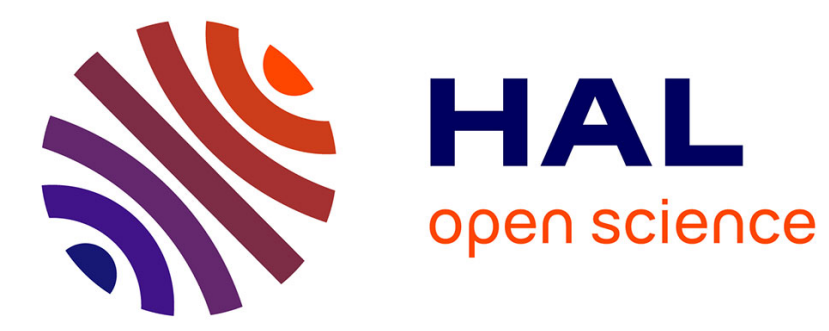

\title{
Study of the Planar Rocking-Block Dynamics With Coulomb Friction: Critical Kinetic Angles
}

\author{
Hongjian Zhang, Bernard Brogliato, Caishan Liu
}

\section{To cite this version:}

Hongjian Zhang, Bernard Brogliato, Caishan Liu. Study of the Planar Rocking-Block Dynamics With Coulomb Friction: Critical Kinetic Angles. Journal of Computational and Nonlinear Dynamics, 2013, 8 (2), pp.11. 10.1115/1.4007056 . hal-00825590

\section{HAL Id: hal-00825590 https://hal.inria.fr/hal-00825590}

Submitted on 3 Nov 2017

HAL is a multi-disciplinary open access archive for the deposit and dissemination of scientific research documents, whether they are published or not. The documents may come from teaching and research institutions in France or abroad, or from public or private research centers.
L'archive ouverte pluridisciplinaire HAL, est destinée au dépôt et à la diffusion de documents scientifiques de niveau recherche, publiés ou non, émanant des établissements d'enseignement et de recherche français ou étrangers, des laboratoires publics ou privés. 
The objective of this paper is to show, through the planar rocking block example, that kinetic angles play a fundamental role in multiple impact with friction. Even in the presence of Coulomb friction, a critical kinetic angle $\theta_{c r}$ is exhibited that allows one to split the blocks into two main classes: slender blocks with a kinetic angle larger than $\theta_{c r}$, and flat blocks with a kinetic angle smaller than $\theta_{c r}$. The value of $\theta_{c r}$ varies with the friction value, but it is independent of the restitution coefficient (normal dissipation). Numerical results are obtained using a multiple impact law recently introduced by the authors. Some comparisons between numerical and experimental results that validate the used model and numerical scheme are presented. However, this paper is mainly based on numerical simulations.

\author{
INRIA Grenoble Rhone-Alpes, Inovallée, \\ 655 avenue de l'Europe \\ 38334 Saint-Ismier, France \\ e-mail: bernard.brogliato@inria.fr \\ Caishan Liu \\ Professor \\ State Key Laboratory for Turbulence \\ and Complex Systems, \\ College of Engineering, \\ Peking University, \\ Beijing 100871, PRC \\ e-mail: liucs@pku.edu.cn
}

\section{Introduction}

Modeling the dynamics of a rigid block hitting a rigid ground has attracted the attention of scientists in the field of Earthquake Engineering for a long time [1-10]. This is also of interest for the study of blocks falling on very steep planes in the mountain [11] and whose trajectories need to be estimated with sufficient accuracy. In parallel, the field of impact dynamics witnessed an intense activity in the past twenty-five years [12-24]. It happens that the problem of modeling impact with friction is a tough issue, especially when there are several simultaneous contact points and when friction is present during the impact (multiple impact with friction). Typically the so-called rocking block problem involves doubleimpact with friction, when one assumes that the base contacts the ground at two points only. Together with chains of balls (Newton cradles), the rocking block is an apparently simple multibody system (the block and the ground); however, it involves multiple impact with friction, and its modeling and dynamical behavior are, consequently, unexpectedly complex. The major drawback of the kinematic restitution laws is that they do not allow the designer to predict the extremely rich dynamical behavior of a block because they model in a very crude way the frictional phenomenon at the impact [14]. The impact dynamics that are used in this paper are based on the Darboux-Keller model studied previously for single impact with friction in two and three dimensions [13,23]. They were extended to the multiple impact case in Refs. [25-29], with and without friction. In these references, many numerical simulation results are shown and compared to experimental results obtained elsewhere on chains of balls [30-32], Newton cradles [33], as well as a bouncing dumbbell [34]. Those comparisons are quite successful, and they demonstrate that the proposed model encapsulates the most important features of multiple impact for rate-independent materials. In particular, both the dissipation (with energetic coefficients of restitution) and the dispersion effects are

${ }^{1}$ Work performed while at INRIA, BIPOP project-team, ZIRST Montbonnot, 655 avenue de l'Europe, 38334 Saint Ismier, France. well reproduced. Detailed and numerous comparisons between numerical and experimental results for the rocking block were obtained elsewhere (see Ref. [35]) and confirm that the used model and numerical scheme provide quite good results.

Since there are too many parameters that may be varied in the planar block/ground system (two restitution coefficients, two-or four-friction coefficients, the elasticity coefficient, the dimensions, the mass, the initial state), it is important to provide a simple guide-line that indicates general patterns for the block motions. It is shown here that the kinetic angle between the constraints plays a significant role. The kinetic angle is uniquely related to the aspect ratio of the block, and it has been well-known for a long time in the rocking block literature that the aspect ratio plays a role in the block dynamics (see Refs. [27,34,36] for results concerning the bouncing dimer). It was also noticed that the kinetic angles are an important parameter in frictionless multiple impact $[13,14,17,37-40]$ because they are closely related to the continuity (or the discontinuity) of the solutions with respect to the initial data. It is intuitively quite clear that slender blocks are less "stable" than flat ones, as they may overturn more easily. From the point of view of the rocking block analysis, the main contribution of this work is to clarify what may be considered as the transition between slenderness and flatness. From the point of view of multiple impact analysis, this work points out that even when friction is present, some critical kinetic angles may be exhibited. The contribution of this paper is, therefore, rooted in two research domains: the rocking block analysis and multiple impact analysis.

This paper is organized as follows: In Secs. 2 and 3 the block dynamics and the kinetic angle definition are briefly recalled. In Sec. 4 the multiple impact law introduced in Refs. $[25-28,36]$ is summarized. Simulation results are presented for the block, in the case with gravity and friction, in Sec. 5. In particular, in Sec. 5 the role played by the kinetic angle between the two unilateral constraints at the impact times is highlighted. Conclusions end the paper in Sec. 6.

\section{The Block Dynamics}

Let us consider the block as a three-degree-of-freedom planar homogeneous solid, with generalized coordinates $q=[x, y, \theta]^{T}$, 


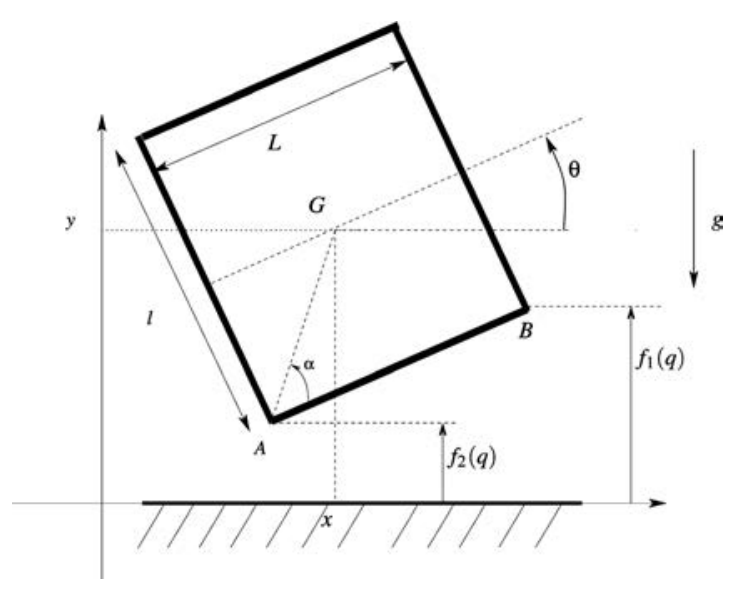

Fig. 1 The planar block

where $x$ and $y$ are the horizontal and vertical coordinates of the center of gravity, $\theta$ is the angular position as depicted in Fig. 1. Following Chapter 6 in Ref. [13], we infer that the block, when $y \leq \sqrt{l^{2}+L^{2}} / 2$, is subject to two unilateral constraints,

$$
\begin{aligned}
& f_{1}(q)=y-\frac{l}{2} \cos (\theta)+\frac{L}{2} \sin (\theta) \geq 0 \\
& f_{2}(q)=y-\frac{l}{2} \cos (\theta)-\frac{L}{2} \sin (\theta) \geq 0
\end{aligned}
$$

where $f_{1}(q) \geq 0$ expresses that point $B$ cannot penetrate into the ground, while $f_{2}(q) \geq 0$ expresses the same for point $A$. The underlying assumption is that the block/ground contact can be represented by the two points $A$ and $B$ at the corners. Obviously, when the block rotates by an angle larger than $\pm \pi / 2$, the contact points change, and one has to reconsider new unilateral constraints (this is what happens when so-called overturn occurs). The admissible domain defined by the unilateral constraints in the $(\theta, y)$ plane is not convex for this choice of generalized coordinates, as illustrated in Fig. 2. One can notice particular angles: the critical angles $\pm \theta_{c}$ at which the curves $f_{1}(q)=f_{2}(q)=0$ in the $(\theta, y)$ plane attain maximum values, and $\theta=0$. The critical angles $\pm \theta_{c}$ correspond to a colinear single impact of the block at $A$ or $B$, respectively, with $A G$ (resp. $B G$ ) colinear to the normal to the ground, and therefore, $\theta_{c}=\pi / 2-\alpha$. At $\theta=0$, one has a

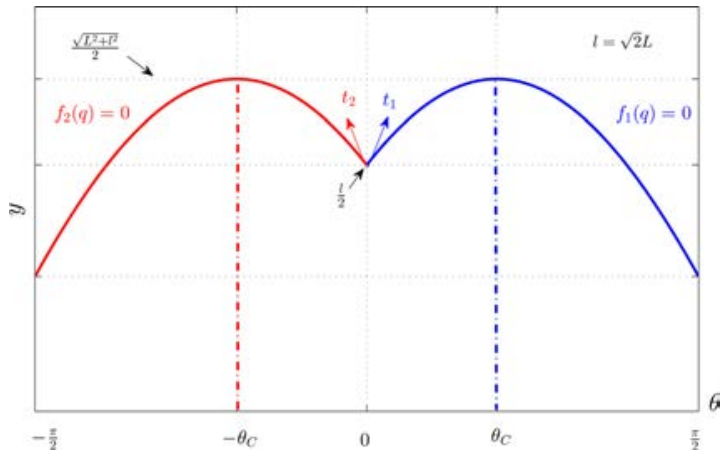

Fig. 2 Admissible domain for $I=\sqrt{2} L$

double-impact ( $A$ and $B$ hit the ground simultaneously), and the admissible domain presents a cusp-singularity.

The dynamics of the block subject to frictionless constraints and gravity are

$$
\begin{aligned}
m \ddot{x}(t) & =0 \\
m \ddot{y}(t)= & \lambda_{n, 1}(t)+\lambda_{n, 2}(t)-m g \\
I_{G} \ddot{\theta}(t)= & \lambda_{n, 1}(t)\left(\frac{l}{2} \sin (\theta(t))+\frac{L}{2} \cos (\theta(t))\right) \\
& +\lambda_{n, 2}(t)\left(\frac{l}{2} \sin (\theta(t))-\frac{L}{2} \cos (\theta(t))\right) \\
0 \leq & \lambda_{n}(t) \perp f(q(t)) \geq 0
\end{aligned}
$$

where the complementarity conditions are componentwise, $f(q)=\left[f_{1}(q), f_{2}(q)\right]^{T}$ and $\lambda_{n}=\left[\lambda_{n, 1}, \lambda_{n, 2}\right]^{T}$. For a block with $G$ at the geometric center, one has $I_{G}=m\left(l^{2}+L^{2}\right) / 12$. In Eq. (2), we have not yet considered the impact with the ground but only those phases of motion where the contact force is a bounded function of time. From Eqs. (1) and (2), the linear complementarity problem (LCP) that allows one to calculate the contact forces during the smooth phases of motion (i.e., outside impact) is given by

$$
0 \leq \lambda_{n}(t) \perp \frac{d^{2}}{d t^{2}} f(q(t))=A(\theta) \lambda_{n}+B(\theta, \dot{\theta}) \geq 0
$$

with

$$
\begin{gathered}
A(\theta)=\left(\begin{array}{cc}
\frac{1}{m}+\frac{1}{4 I_{G}}(l \sin (\theta)+L \cos (\theta))^{2} & \frac{1}{m}+\frac{1}{4 I_{G}}\left(l^{2} \sin ^{2}(\theta)-L^{2} \cos ^{2}(\theta)\right) \\
\frac{1}{m}+\frac{1}{4 I_{G}}\left(l^{2} \sin ^{2}(\theta)-L^{2} \cos ^{2}(\theta)\right) & \frac{1}{m}+\frac{1}{4 I_{G}}(l \sin (\theta)-L \cos (\theta))^{2}
\end{array}\right) \\
B(\theta, \dot{\theta})=\left(\begin{array}{l}
-g+\frac{1}{2} \dot{\theta}^{2}(l \cos (\theta)-L \sin (\theta)) \\
-g+\frac{1}{2} \dot{\theta}^{2}(l \cos (\theta)+L \sin (\theta))
\end{array}\right)
\end{gathered}
$$

One has $A(\theta)=A^{T}(\theta)$, and one easily checks that $A(\theta)$ is positive definite except at $\theta= \pm \pi / 2$. These values are, however, outside the range of block orientations within which the analysis is done. We conclude that for all angles $\theta \in(-\pi / 2, \pi / 2) A(\theta)>0$, and the normal contact force $\lambda_{n}$ can be computed uniquely as the solution of the LCP in Eq. (3) whatever $\theta$ and $\dot{\theta}$. $A(\theta)$ is the so-called Delassus matrix of the system in Eqs. (1) and (2). When Coulomb friction acts at the contact points, the dynamics become 


$$
\begin{aligned}
m \ddot{x}(t)= & \lambda_{t, 1}(t)+\lambda_{t, 2}(t) \\
m \ddot{y}(t)= & \lambda_{n, 1}(t)+\lambda_{n, 2}(t)-m g \\
I_{G} \ddot{\theta}(t)= & \lambda_{n, 1}(t)\left(\frac{l}{2} \sin (\theta(t))+\frac{L}{2} \cos (\theta(t))\right)+\lambda_{n, 2}(t)\left(\frac{l}{2} \sin (\theta(t))-\frac{L}{2} \cos (\theta(t))\right) \\
& \quad\left(\frac{l}{2} \cos (\theta)-\frac{L}{2} \sin (\theta)\right) \lambda_{t, 1}+\left(\frac{l}{2} \cos (\theta)+\frac{L}{2} \sin (\theta)\right) \lambda_{t, 2} \\
0 \leq \lambda_{n}(t) \perp f(q(t)) \geq 0 & \\
\lambda_{t, i}(t) \in & -\mu_{i} \lambda_{n, i}(t) \operatorname{sgn}\left(v_{t, i}(t)\right), i=1,2
\end{aligned}
$$

where $\mu_{i}>0$ is the friction coefficient at contact $i$, and $v_{t, i}$ is the tangential velocity at the point $i$, i.e., $v_{t, 1}=\dot{x}+(l / 2 \cos (\theta)$ $+L / 2 \sin (\theta)) \dot{\theta}$ at $B$ and $v_{t, 2}=\dot{x}+(l / 2 \cos (\theta)-L / 2 \sin (\theta)) \dot{\theta}$ at $A$ (from which $v_{t, 1}=v_{t, 2}$ when $\theta=0$ ). Notice that if the contact point $i$ detaches then the complementarity conditions imply that $\lambda_{n, i}=0$ so $\lambda_{t, i}=0$. The dynamics in Eq. (4) stand for fixed ground. It is possible to rewrite compactly Eqs. (2) and (4) as

$$
M \ddot{q}(t)=W_{n}(q(t)) \lambda_{n}(t)+W_{t}(q(t)) \lambda_{t}(t)-\mathbf{g}
$$

with $\mathbf{g}=[0 m g 0]^{T}, M=\operatorname{diag}\left(m, m, I_{G}\right), W_{n}$ and $W_{t}$ are easily identified from Eqs. (2) and (4).

\section{Kinetic Angle}

The kinetic angle between two unilateral constraints is a quantity, which reflects the couplings that exist between the normal directions to the constraints boundaries and the inertial properties of the system. Kinetic angles are known to play an important role in frictionless multiple impact [13,17,37-39]. In particular, it is known that the value $\pi / 2$ is a critical value of the kinetic angle. Roughly speaking, trajectories are continuous with respect to initial data for kinetic angles $\leq \pi / 2$ and discontinuous for kinetic angles $>\pi / 2$, depending on the restitution coefficients [39]. Kinetic angles are calculated in the kinetic metric, that is the metric defined from the inertia matrix (supposed to be symmetric, positive definite). The kinetic angle between the two unilateral constraints at the impact times $(\theta(t)=0)$ is given $b^{2}$

$$
\theta_{12}=\pi-\arccos \frac{\nabla f_{1}(q)^{T} M^{-1}(q) \nabla f_{2}(q)}{\sqrt{\nabla f_{1}(q)^{T} M^{-1}(q) \nabla f_{1}(q)} \sqrt{\nabla f_{2}(q)^{T} M^{-1}(q) \nabla f_{2}(q)}}
$$

Starting from the frictionless impact dynamics of a Lagrangian system with mass matrix $M(q) \in \mathbb{R}^{n \times n}$ and a vector of unilateral constraints $f(q) \geq 0$ with $f(q) \in \mathbb{R}^{m}$, one finds [13]

$$
M(q) \cdot\left(\dot{q}\left(t^{+}\right)-\dot{q}\left(t^{-}\right)\right)=\nabla f(q) P(t)
$$

where $P(t) \in \mathbb{R}^{m}$ is the magnitude of the impact pulse at the impact time $t$, so that

$$
\nabla f^{T}(q) \cdot\left(\dot{q}\left(t^{+}\right)-\dot{q}\left(t^{-}\right)\right)=\nabla f^{T}(q) M^{-1}(q) \nabla f(q) P(t)
$$

where the time argument of $q$ in $M(q)$ and $f(q)$ is omitted in Eqs. (7) and (8) to lighten the notation. The $m \times m$ matrix $\nabla f^{T}(q) M^{-1}(q) \nabla f(q)$ is the so-called Delassus matrix of the unilaterally constrained Lagrangian system, whose components are given by $\nabla f_{i}^{T}(q) M^{-1}(q) \nabla f_{j}(q), 1 \leq i \leq m$, and $1 \leq j \leq m$. This

\footnotetext{
${ }^{2}$ The kinetic angle is obtained after subtraction from $\pi$ because the normal vectors point outside the admissible domain of the configuration space.
}

shows that first the kinetic angles naturally appear in multiple impact phenomena and second that when all the constraints are pairwise orthogonal in the kinetic metric then the Delassus matrix is diagonal, and the impacts may be considered as being decoupled. Otherwise, couplings exist, and it may be expected that the impact process is more involved. For the rocking block, the kinetic angle $\theta_{12}$ between the two constraints is given by

$$
\theta_{12}=\pi-\arccos \left(\frac{l^{2}-2 L^{2}}{l^{2}+4 L^{2}}\right)
$$

at $\theta=0$. Denoting the aspect ratio as $a \triangleq l / L$, we may rewrite it as $\theta_{12}=\pi-\arccos \left(\left(a^{2}-2\right) /\left(a^{2}+4\right)\right)$ : there is a one-to-one correspondence between $a$ and $\theta_{12}$. It satisfies $\theta_{12}=\pi / 2$ if $l=\sqrt{2} L$, $0<\theta_{12}<\pi / 2$ if $0<l<\sqrt{2} L$ (flat block), and $\pi>\theta_{12}>\pi / 2$ if $l>\sqrt{2} L$ (slender block). When $a$ varies from 0 (infinitely flat block with infinite width $L$ ) to $+\infty$ (infinitely slender block with infinite height $l$ ) then $\theta_{12}$ varies from $\pi / 4$ to $\pi$. The fact that $\theta_{12} \in[\pi / 4, \pi]$ means that one expects that the block/ground system possess rich dynamics and may serve as a nice example of multiple impact with friction. The interest of studying the block dynamics as a function of the kinetic angle between the two boundaries at $\theta=0$ is that it allows us to determine that a block is not of the slender type just if $l>L$. It is clear that since there is a oneto-one relation between the kinetic angle and the aspect ratio, one could use equivalently the aspect ratio, which is a familiar quantity in the Earthquake Engineering literature. However the kinetic angle is a more general notion that can be used in any type of multiple impact in multibody systems with unilateral constraints (like chains of balls), whereas the aspect ratio is attached to planar blocks. Moreover, one might lead a more general analysis than the one in this paper by considering non symmetric blocks, in which case it could be useful to keep the inertial parameters like the mass and the moment of inertia $I_{G}$ in the kinetic angle expression. Considering the kinetic angles rather than the aspect ratio, therefore, paves the way towards more general analysis for more complex blocks (and grounds).

Remark 1. In Refs. [27,34,36], the dynamics of a planar bouncing dimer are studied. The dimer is made of two identical spheres with radius $R$ connected by a rigid rod with length $L$. Using the same notations as for the block, the two unilateral constraints for the dimer are $f_{1}(q)=y+(L / 2+R) \sin (\theta)-R \geq 0$ and $f_{2}(q)=y$ $-(L / 2+R) \sin (\theta)-R \geq 0$. Some calculations yield that the kinetic angle between the two constraints at $\theta=0$ (double impact), and with all masses equal to 1 for simplicity, is given by $\theta_{12}=\pi$ $-\arccos ((1 / 3-\alpha) /(1 / 3+\alpha))$ with $\alpha=(1+2 a)^{2} /\left(16 a^{2} / 5+1 / 3\right.$ $\left.\left.+2(1+2 a)^{2}\right)\right), a=R / L$. The flattest dimer has $L=+\infty$, and the less flat one has $L=0$ (the two balls are stuck together). The two kinetic angle values that correspond to these extreme cases are $\theta_{12}=\pi-\arccos (-1 / 8) \approx 1.445 \mathrm{rad}$ and $\theta_{12}=\pi-\arccos (-1 / 29)$ $\approx 1.536 \mathrm{rad}$, which are both slightly smaller than $\pi / 2 \approx 1.571 \mathrm{rad}$. This means that the dimer and the block, despite their apparent 
similarity, possess different dynamical behaviors in the sense that the dimer kinetic angle varies little and never exceeds $\pi / 2$ (the dimer is always flat), while the block kinetic angle may vary much more. Finally, let us point out that chains of balls are also a quite interesting system with multiple impact. However, friction is usually not considered in Newton cradle, whereas waves effect plays a significant role. Consider a linear chain of three aligned balls with coordinates $q_{1}, q_{2}, q_{3}$, radii $R$, and masses $m_{1}, m_{2}, m_{3}$. There are two unilateral constraints $f_{1}(q)=q_{2}-q_{1}+2 R \geq 0$ and $f_{2}(q)=q_{3}-q_{2}+2 R \geq 0$. The kinetic angle between them is given by $\left.\quad \theta_{12}=\arccos \left(\sqrt{m_{1} m_{3}} / \sqrt{\left(m_{1}+m_{2}\right)\left(m_{2}+m_{3}\right)}\right)\right)$. If $m_{1}=m_{2}=m_{3}$ then $\theta_{12}=\arccos 1 / 2=\pi / 3$. In fact, $\theta_{12} \in[0, \pi / 2]$ if $m_{1}$ and $m_{3}$ are much larger than $m_{2}$ then $\theta_{12}$ approaches $0 \mathrm{rad}$, whereas if $m_{2}$ is much larger than $m_{1}$ and $m_{3}$, it approaches $\pi / 2$. Clearly the block/ground, chains of balls, and boucing dimer are systems involving multiple impact of different kinds, and they deserve separate studies.

Rocking Motions. The rocking motion is a widely used term in the Earthquake Engineering literature. Rocking refers to a macroscopic behavior of the block that performs some kind of oscillations like an inverted pendulum while rotating alternatively around each corner $A$ and $B$. In other words, the angle $\theta$ takes alternatively positive and negative values. However, rocking may occur with the contact points that stick in both normal and tangential directions (perfect rocking), just in the normal direction while tangential slip is occurring, or with sequences of impacts at the impacting corner. When $\theta$ takes only positive (or only negative) values, one speaks of half-rocking. Then one corner point always keeps the contact with the ground while the other one rebounds.

\section{The Extended Darboux-Keller Impact Dynamics}

The impact model proposed in Refs. [25-28] is summarized in this section. The contact stiffnesses are denoted as $k_{i}$, the potential energy at contact $i$ is $E_{i}, \eta$ is the elasticity coefficient. The matrix $W_{n}(q)$ is the Jacobian between the generalized velocities $\dot{q}$ and the contact points normal relative velocities, i.e., $W_{\mathrm{n}}^{T}(q)=\partial f / \partial q$ $=\nabla f^{T}(q) \in \mathbb{R}^{2 \times 3}$, whereas $W_{n}(q) \lambda_{n}$ represents the generalized contact force associated to the generalized coordinates $q$ (see Eq. (2)). $P_{n, i}$ denotes the interaction normal force impulse at contact point $i$, and $P_{n}=\left[P_{n, 1}, P_{n, 2}\right]^{T}$ is the vector of normal impulses. The mass matrix $M \in \mathbb{R}^{3 \times 3}$ is given by $M=\operatorname{diag}\left(m, m, I_{G}\right)$. An important assumption in this model, which is an extension of the Darboux-Keller approach [13], is that positions $q$ are constant during the impact process. Thus, $W_{n}(q)$ is constant and equal to its initial value at the beginning of the collision process. For this reason, it is denoted simply as $W_{n}$. The vectors $w_{n, j}$ are the columns of $W_{n}$. In the frictionless case, one obtains:

(1) Contact parameters: $\gamma_{i j}=k_{i} / k_{j}$ (stiffnesses ratios), $e_{n, j}^{*}$ (energetic restitution coefficients), $1 \leq i \leq 2,1 \leq j \leq 2, \eta$ $\left(=1\right.$ for linear elasticity, $=\frac{3}{2}$ for Hertz contact, or other values).

(2) Dynamical equation:

$$
M \frac{d \dot{q}}{d P_{n, i}}=W_{n} \frac{d P_{n}}{d P_{n, i}} \quad \text { if } \quad E_{j i}\left(P_{n, j}, P_{n, i}\right) \leq 1 \quad \text { for } \quad j \neq i
$$

where the potential energy ratios are defined as

$$
E_{j i}\left(P_{n, j}, P_{n, i}\right)=\frac{E_{j}\left(P_{n, j}\right)}{E_{i}\left(P_{n, i}\right)}, \quad 1 \leq i \leq 2,1 \leq j \leq 2
$$

and the potential energy at contact $j$ as

$$
E_{j}\left(P_{n, j}\right)=\int_{0}^{P_{n, j}(t)} w_{n, j}^{T} \dot{q} d P_{n, j}
$$

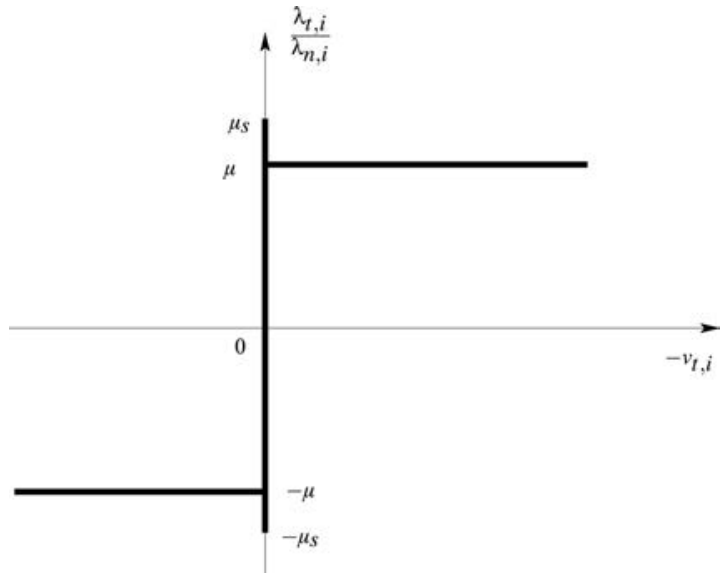

Fig. 3 The friction model

(3) Distributing law ${ }^{3}$ :

$$
\frac{d P_{n, j}}{d P_{n, i}}=\gamma_{j i}^{\frac{1}{\eta+1}}\left(E_{j i}\left(P_{n, j}, P_{n, i}\right)\right)^{\frac{\eta}{\eta+1}}
$$

The flexibilities and the plasticity/damage effects are taken into account by a bi-stiffness model of contact, and it is possible that several compression/expansion phases occur at the same contact point $j$. The times $t_{c}$ of maximal compression at the contact $j$ are calculated from $\dot{\delta}_{j}\left(t_{c}\right)=0$, where $\delta_{j}$ is the relative normal displacement at contact $j\left(\dot{\delta}=W_{n}^{T} \dot{q}=\nabla f^{T}(q) \dot{q}\right.$, i.e., $\left.\dot{\delta}_{j}=w_{j}^{T} \dot{q}\right)$. The termination time $t_{f}$ is calculated from the energy constraint $\mathcal{W}_{e, j}=-\left(e_{n, j}^{*}\right)^{2} \mathcal{W}_{c, j}$, where the works during compression and expansion phases are given by, respectively,

$$
\mathcal{W}_{c, j}=\int_{0}^{P_{n, j}\left(t_{c}\right)} w_{n, j}^{T} \dot{q} d P_{n, j}, \mathcal{W}_{e, j}=\int_{P_{n, j}\left(t_{c}\right)}^{P_{j}\left(t_{f}\right)} w_{n, j}^{T} \dot{q} d P_{n, j}
$$

In Eq. (10), the impulse $P_{n, i}$ at contact $i$ is the so-called principal impulse that is chosen as the new time-variable in the impact model. Contrarily to the single impact case, it may change during the impact process when multiple impact is considered (see Ref. [25] for details). In view of Eq. (2) in the frictionless case, the impact dynamics reduce to a two-dimensional system because $d \dot{x} / d P_{n, i}=0$. Coulomb friction can be easily added in the impact model at the force (or infinitesimal impulse) level. In such a case, the right-handside of Eq. (10) has to be modified accordingly with the insertion of the tangential force components (see Eqs. (4) and (5)),

$$
M \frac{d \dot{q}}{d P_{n, i}}=W_{n} \frac{d P_{n}}{d P_{n, i}}+W_{t} \frac{d P_{t}}{d P_{n, i}} \quad \text { if } \quad E_{j i}\left(P_{n, j}, P_{n, i}\right) \leq 1 \text { for } j \neq i
$$

In this work, we use an enhanced model of friction with a static $\mu_{s}$ and a dynamic $\mu$ friction coefficients as depicted in Fig. 3, both during and outside the impacts. This impact model is, therefore, a rigid body model that incorporates some flexibility effects, with one restitution coefficient and one (or two) friction coefficient per contact. More details on the implementation may be found in Refs. [28,36], and the code, which has been used for the simulation, is given in Ref. [35] (a similar eventdriven code is also available in the Siconos platform [41]). For the dynamics outside the impact, the LCP in Eq. (3) is used to

\footnotetext{
${ }^{3}$ The power of the potential energy ratio $E_{j i}\left(P_{n, j}, P_{n, i}\right)$ is inverted in Refs. [25,26].
} 

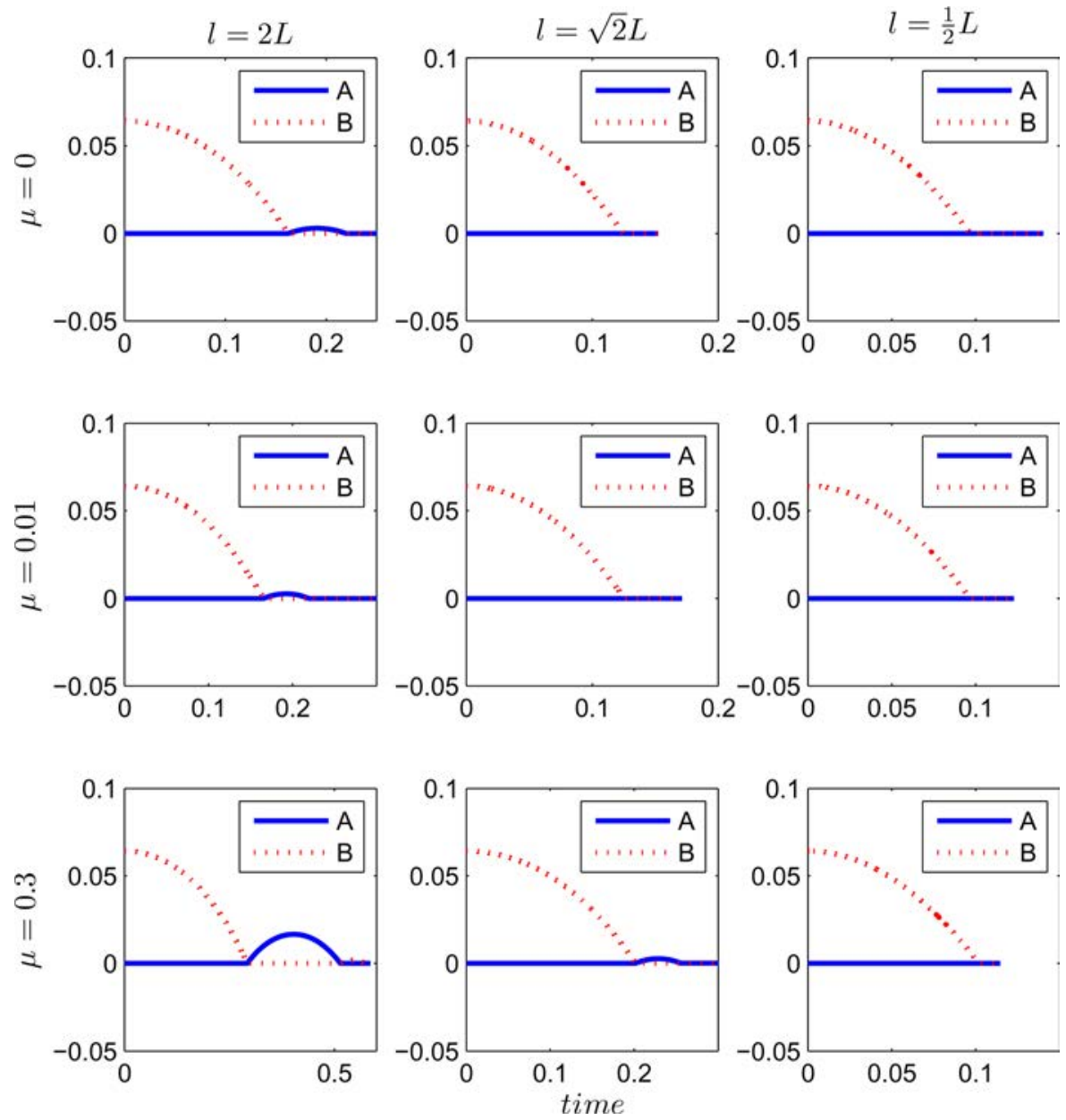

Fig. $4 y_{A}(t)$ and $y_{B}(t)$, with $e_{n, 1}^{*}=e_{n, 2}^{*}=0$, varying $\mu$

integrate the system, and either explicit Euler or Runge-Kutta algorithms are implemented. This is not the optimal way to integrate such complementarity systems [37] but proves to be sufficient for this application. It is noteworthy that the numerical scheme that is employed next is of the event driven type [37] and that all the stick/slip and contact/detachment conditions are carefully taken care of.

\section{Impact With Coulomb Friction: Study of Critical Kinetic Angle}

Let us now turn our attention to the case of impact and contact with friction. In our model, we introduce a static $\mu_{s}$ and a dynamic $\mu$ coefficients of friction as shown in Fig. 3. We first consider three values of $\theta_{12}$, i.e., the ratios $l / L=2, \sqrt{2}$, and $1 / 2$. Gravity is considered in the simulation, $m=0.02 \mathrm{~kg}$, and the ground is supposed to be fixed. The initial data are a positive angle $\theta(0)$ and zero velocity. In a second stage, we exhibit the variation of a critical kinetic angle (equal to $\pi / 2$ in the frictionless case) when the friction and the normal restitution are varied. Surprisingly enough, friction does not alter the global picture of the double-impact process.

The dynamic and the static friction coefficients are varied: $\left(\mu_{s}, \mu\right)=(0,0),(0.02,0.01)$, and $(0.5,0.3)$. The following comments arise from Figs. 4 and 5 where it is considered two different normal dissipations:

(1) $e_{n, 1}^{*}=e_{n, 2}^{*}=0$ in Fig. 4: When $\theta_{12}<\pi / 2(l=L / 2)$, point $A$ never rebounds, $B$ hits the ground once, and the block is at rest. When $\theta_{12}>\pi / 2(l=2 L), A$ always rebounds a little, the rebound at $A$ is bigger for larger $\mu$, the block is at rest af- ter one impact at $B$ followed by one impact at $A$. The case $\theta_{12}=\pi / 2(l=\sqrt{2} L)$ makes a transition between both cases.

(2) $e_{n, 1}^{*}=e_{n, 2}^{*}=0.8$ in Fig. 5: When $\theta_{12}=\pi / 2$ and $\mu$ is small or zero, $A$ does not detach from the ground, and $B$ undergoes a series of impacts (half-rocking motion, with no exchange of energy from $B$ to $A$ ); $A$ detaches and collides also (i.e., part of the energy is exchanged between $A$ and $B$ ) when $\mu$ is large. When $\theta_{12}<\pi / 2$ and $\mu$ is small, there is a strong exchange of energy from $B$ to $A$ at the first impact and from $A$ to $B$ at the second impact; then the energy seems to be balanced between the two contact points; for large $\mu$ both contact points take similar energies. When $\theta_{12}>\pi / 2$ and $\mu$ is small, the first rebound at $B$ is much larger that the ones at $A$, indicating that the energy exchange between $B$ and $A$ exists but is small; when $\mu$ gets larger, a much larger part of the energy transfers from $B$ to $A$ at the first impact, and subsequently, the energy is more dispersed at the two contact points. Obviously $\theta_{12}=\pi / 2$ makes a transition between two different mechanisms of energy dispersion in the block.

(3) The energy dispersion is such that rocking or half-rocking seldom appears (just as a pathological case for $\theta_{12}=\pi / 2$ in Fig. 5) for the chosen parameters values. It is apparent from Fig. 5 that the energy dispersion may be a complex nonlinear phenomenon, even for such a simple system as the planar block with two contact points and a fixed base.

One infers from these numerical results that the block behavior depends on the kinetic angle $\theta_{12}$ value, and that the value $\theta_{12}=\pi / 2$ seems to define an intermediate kinetic angle between flat and slender blocks. The next results refine this analysis. 

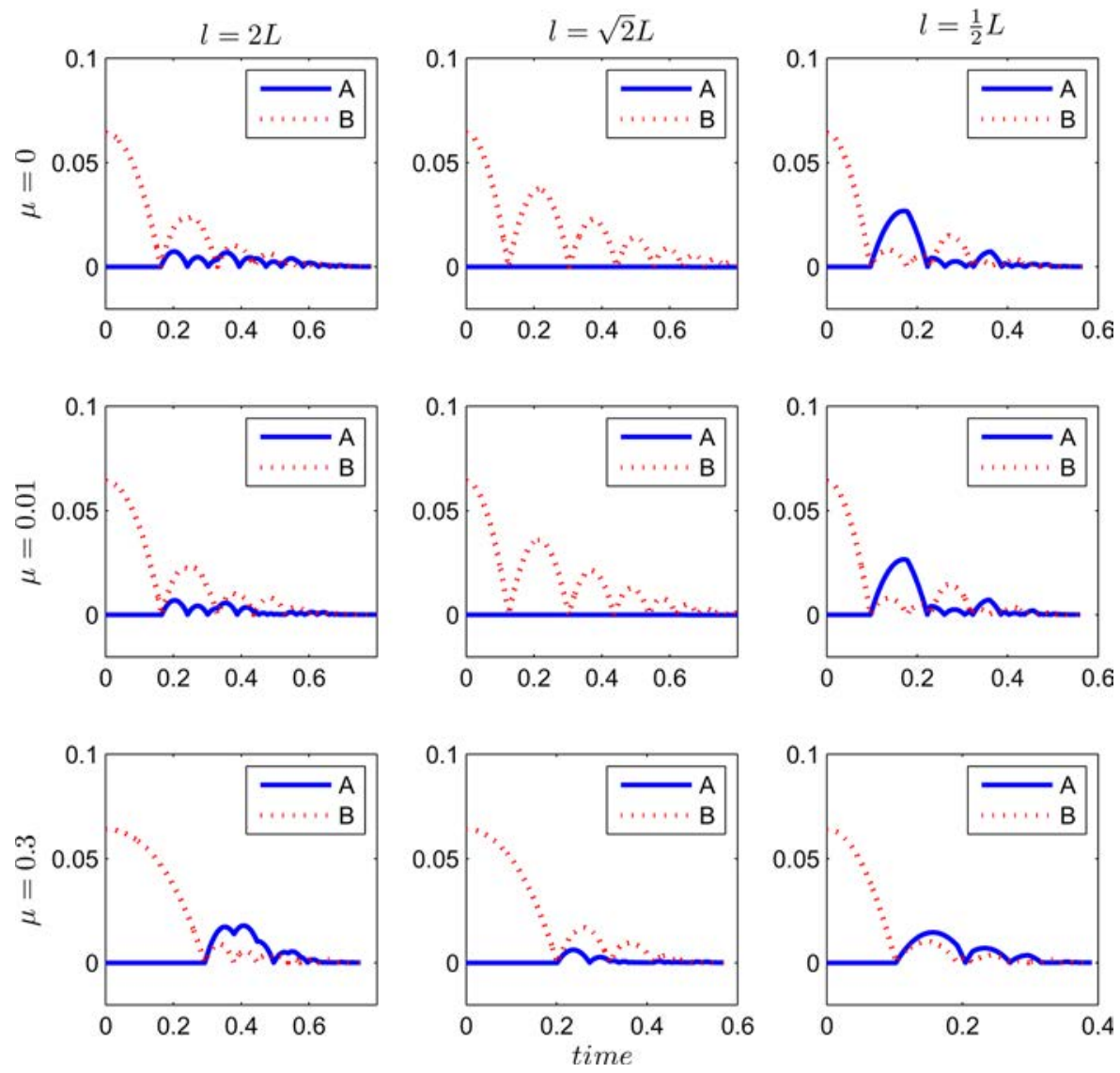

Fig. $5 y_{A}(t)$ and $y_{B}(t)$, with $e_{n, 1}^{*}=e_{n, 2}^{*}=0.8$, varying $\mu$

Remark 2. At first sight, it seems that $e_{n, 1}^{*}=e_{n, 2}^{*}=0$ is a necessary choice to model perfect rocking motion, i.e., rocking where the corner that hits the ground sticks on it after the impact. Perfect rocking is an idealization, and rocking usually is performed only approximately: rebounds and slip occur at the impacting corner before it sticks. Experimental results in the literature report rocking motions with $e_{n, i}^{*}=0.9[3,42,43]$. One can try to use a kinematic restitution model to simulate approximate rocking with $e_{n, i}>0$. The major issue is then to correctly handle Coulomb friction during the rebound phase, in particular at the impact (see Ref. [14]). The Darboux-Keller approach with energetic restitution coefficients allows one to correctly model impact with friction.

It is clear that the aspect ratio, which defines the kinetic angle, plays a significant role in the free-rocking motion. Let us now investigate more deeply the kinetic angle influence by studying the ratio of the vertical velocities $d \triangleq \dot{y}_{A}\left(t^{+}\right) / \dot{y}_{B}\left(t^{-}\right)$after an impact at $B$ while the block rotates around $A$. This ratio provides an estimation on how much "energy" is transferred from $B$ to $A$, which may be thought of as a form of energy dispersion in the block. It is noteworthy that the first impact is the only two-impact, since the rebound phase that may follow involves simple impacts only. Here, we study the dependence of $d$ on the aspect ratio, the friction, and the restitution, i.e., $d\left(e_{n}^{*}, l / L, \mu, \mu_{s}\right)$. In order to separate the effect of dissipation from the other effects we first focus on $d\left(1, l / L, \mu, \mu_{s}\right)$ in Figs. $6(a)-9(b)$. In Figs. $6(a)-6(f)$, the curves $\left(\frac{l}{L}, d\left(1, l / L, \mu, \mu_{s}\right)\right)$ are depicted for various values of $\mu$ and $\mu_{s}$. The dashed line on each figure represents the value of $\cos \left(\theta_{12}\right)$. The initial data are zero velocity and $\theta(0)=0.12 \mathrm{rad}$, and $m=2$ $\mathrm{kg}$. In all the simulation process, $L=0.24 \mathrm{~m}$ and $l$ is varied. Some comments arise:

(1) The $\left(l / L, d\left(1, l / L, \mu, \mu_{s}\right)\right.$-curves all possess a similar $\mathrm{V}$-shape, independently of the friction value.
(2) The minimum is attained at $\theta_{12}=\pi / 2$ for $\mu=\mu_{s}=0$, Fig. $6(a)$. Then, in proportion as friction increases, the minimum point moves to the left until it reaches a stationary point as illustrated in Figs. 6(a)-6(f). In other words, there is a "critical" kinetic angle $\theta_{c r}$ that moves from $\theta_{c r}=\pi / 2$ in Fig. $6(a)$ to $\theta_{c r} \approx \pi / 2.5(\approx 70 \mathrm{deg})$ for $l / L \approx 0.75$ in Fig. $6(f)$. The stationarity of $\theta_{c r}$ after it has reached $\pi / 2.5$ is explained by the fact that once $\mu$ and $\mu_{s}$ are large enough, the contact/impact points always stick (no slip modes exist) on the right of the critical aspect ratio (while on the left stick/slip still exists as depicted in Figs. 8(a) and 8(b)), and increasing the friction does not change the dynamics.

(3) The minimum value $\theta_{c r}=\pi / 2.5$ is attained for the aspect ratio $l / L=0.75>\sqrt{2} / 2=0.7071$. For the friction values indicated in Fig. $6(f)$, the contact points slip after the impact for $\sqrt{2} / 2 \leq l / L<1$ and stick for $l / L \geq 1$ (see also Figs. $8(a)$ and $8(b)$ ). If friction continues to increase, then the aspect ratio for the minimum attains asymptotically $\sqrt{2} / 2$. For such very large values of the friction, the contact points are always sticking (outside and during the impact) in the region $l / L>\sqrt{2} / 2$, as illustrated in Figs. $8(a)$ and $8(b)$. Interestingly enough, this value is found to be a characteristic aspect ratio when considering a kinematic restitution law with angular velocity restitution coefficient $r=\dot{\theta}\left(t^{+}\right) / \dot{\theta}\left(t^{-}\right)$and sticking contact points (see Figs. 3 and 5 in Ref. [14]). The perfectly sticking contact/impact points correspond to the fundamental Housner assumption for rocking motion [2].

(4) For the parameter values of Fig. 6(f), the block motion is rocking for $l / L>1$ and half-rocking for smaller values. The half-rocking motion with $l / L=1$ is experimentally confirmed to exist in Ref. [3]. This is once again related to the results in Ref. [14], which show that the Housner 


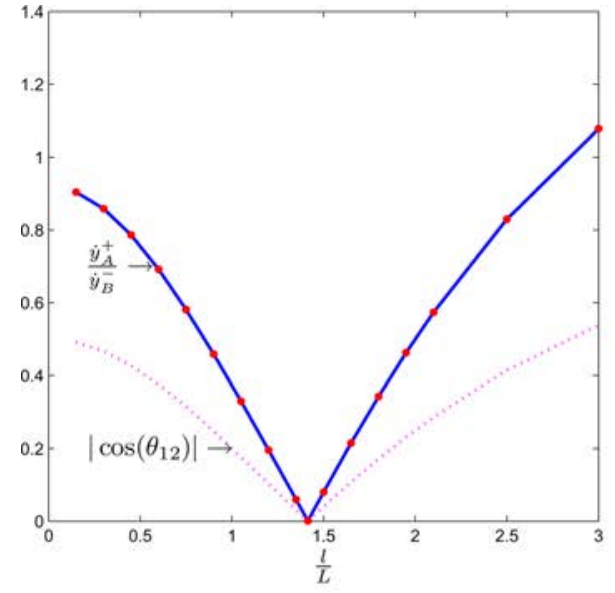

(a) $\mu=\mu_{s}=0, e_{n}^{*}=1$

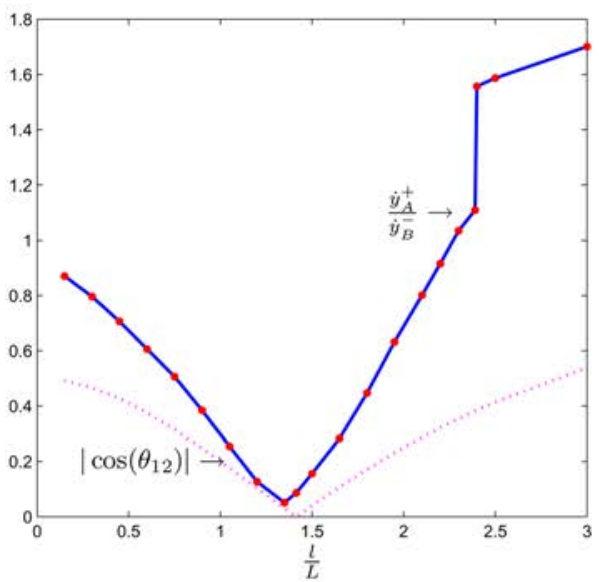

(c) $\mu=0.15, \mu_{s}=0.3, e_{n}^{*}=1$

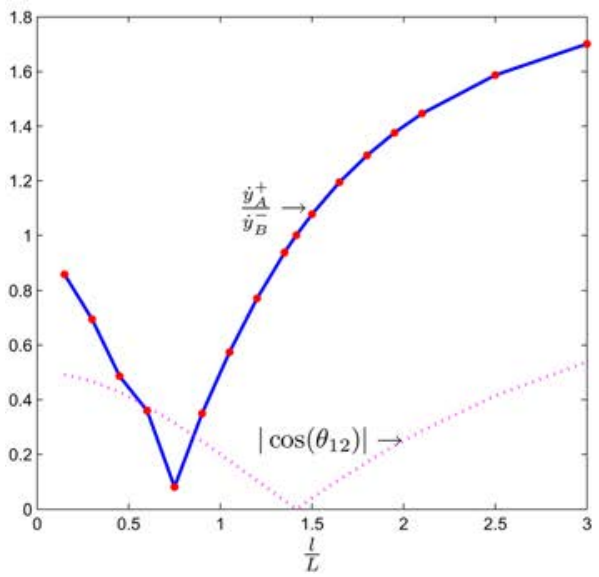

(e) $\mu=0.45, \mu_{s}=0.9, e_{n}^{*}=1$

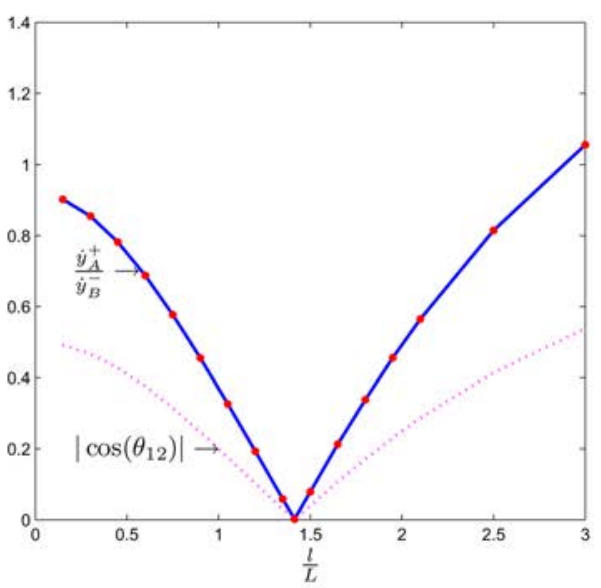

(b) $\mu=0.01, \mu_{s}=0.02, e_{n}^{*}=1$

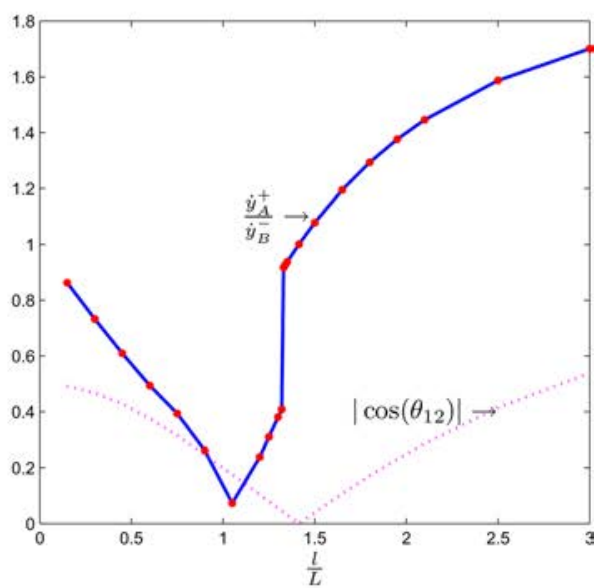

(d) $\mu=0.3, \mu_{s}=0.5, e_{n}^{*}=1$

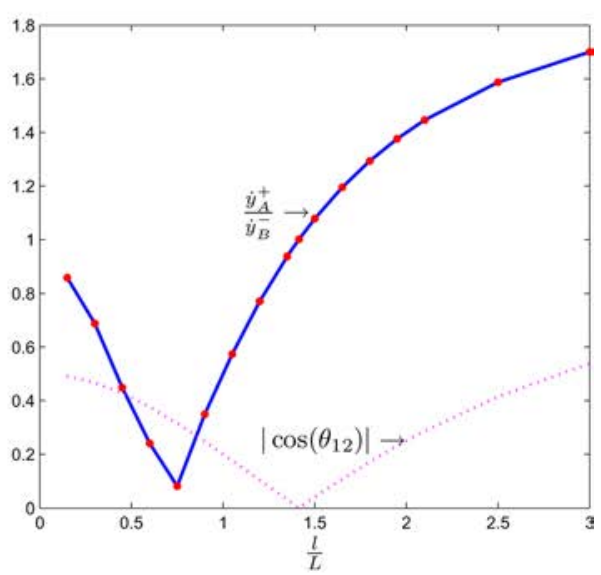

(f) $\mu=0.6, \mu_{s}=1, e_{n}^{*}=1$

Fig. 6 Dispersion $d$ with varying friction

assumptions imply that rocking can be described only for $l / L \geq \sqrt{2} / 2$.

(5) Our analysis with the LZB model, therefore, confirms that the all-sticking assumption is valid only for $l / L>\sqrt{2} / 2$ and high enough friction because otherwise slipping phases may exist for flat blocks as shown in Figs. 8(a) and $8(b)$. It also shows that the all-sticking assumption corresponds to unrealistic values of the friction, and is met in practice only if the block is slender enough.
(6) One sees in Figs. 7(a) and 7(b) that, in the frictionless case, there is a simple and remarkable relation between $d(1, l / L, 0,0)$ and $\cos \left(\theta_{12}\right)$. This is, however, lost when friction is present.

(7) The $\theta(t)$ response is depicted in Figs. $9(a)$ and $9(b)$ for fixed friction $\mu=0.6, \mu_{s}=1$ (hence stick always occurs) and varying aspect ratio $l / L$. After the first double-impact, the block becomes airborne before it collides again with the ground. 


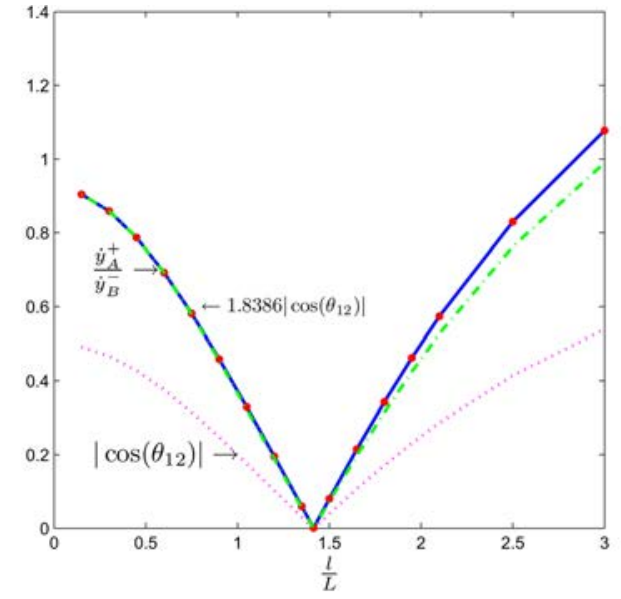

(a) Matching with $1.8386\left|\cos \left(\theta_{12}\right)\right|$ for $l / L<\sqrt{2}$

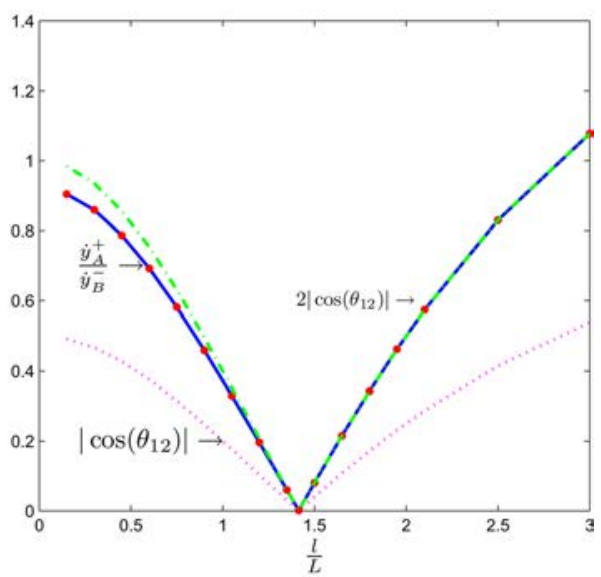

(b) Matching with $2\left|\cos \left(\theta_{12}\right)\right|$ for $l / L>\sqrt{2}$

Fig. 7 Dispersion $d$ with $\mu=\mu_{s}=0, e_{n}^{*}=1$

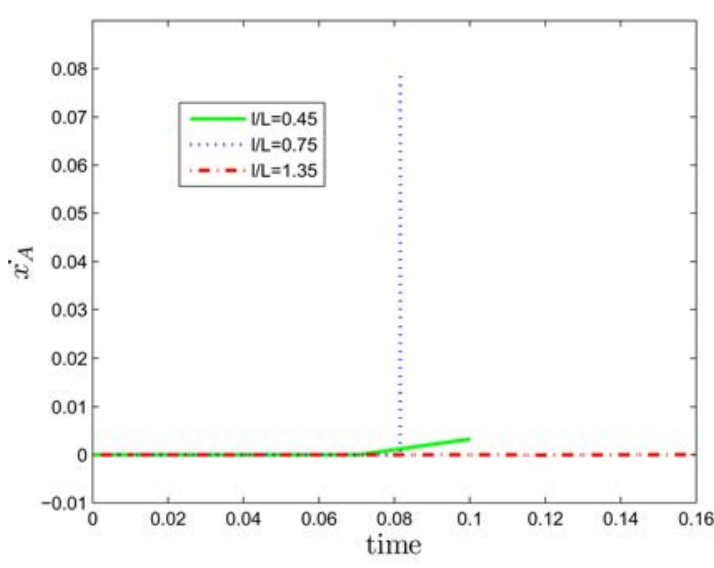

(a) Velocities before and after the impact

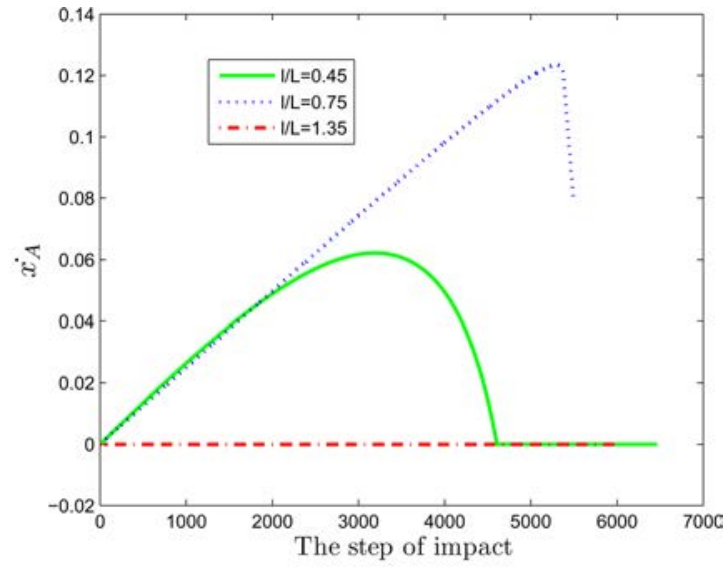

(b) Velocities during the impact

Fig. 8 Stick/slip behavior at $A, \mu=0.6, \mu_{s}=1, e_{n}^{*}=1$

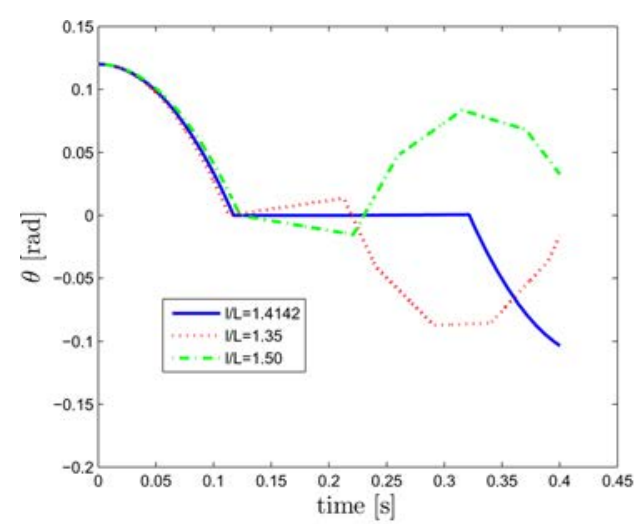

(a) $\frac{l}{L}=1.4142,1.35,1.50$

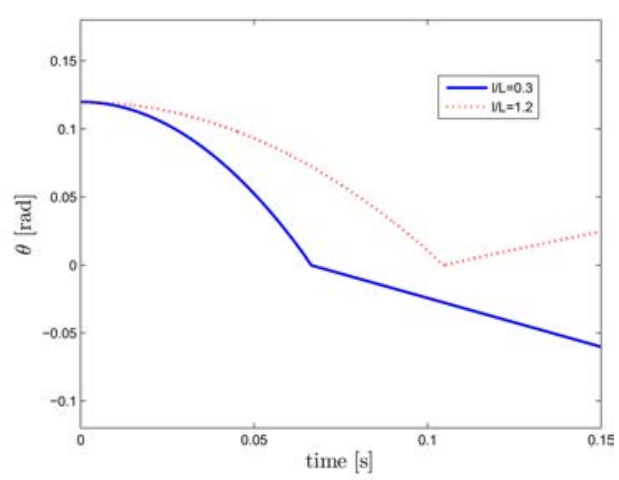

(b) $\frac{l}{L}=0.3,1.2$

Fig. $9 \theta(t)$ response, $\mu=0.6, \mu_{s}=1, e_{n}^{*}=1$

(8) When friction is present but small enough, there exist slip and stick phases of motion. This creates some unstable behaviors as seen on Figs. 6(c) and 6(d) with a jump in $d\left(1, l / L, \mu, \mu_{s}\right)$ at some $l / L$. This jump shrinks when friction is zero and when friction is large enough. In the first case, it shrinks in the asymptotic part on the right of the curve. In the second case, it shrinks in the critical minimum point.

(9) Comparing Figs. 8(a) and 8(b) and Figs. 11(a) and 11(b), one infers that increasing the friction increases the sticking phases and decreases the slipping phases, as expected. However this is true for sufficiently slender blocks only, 


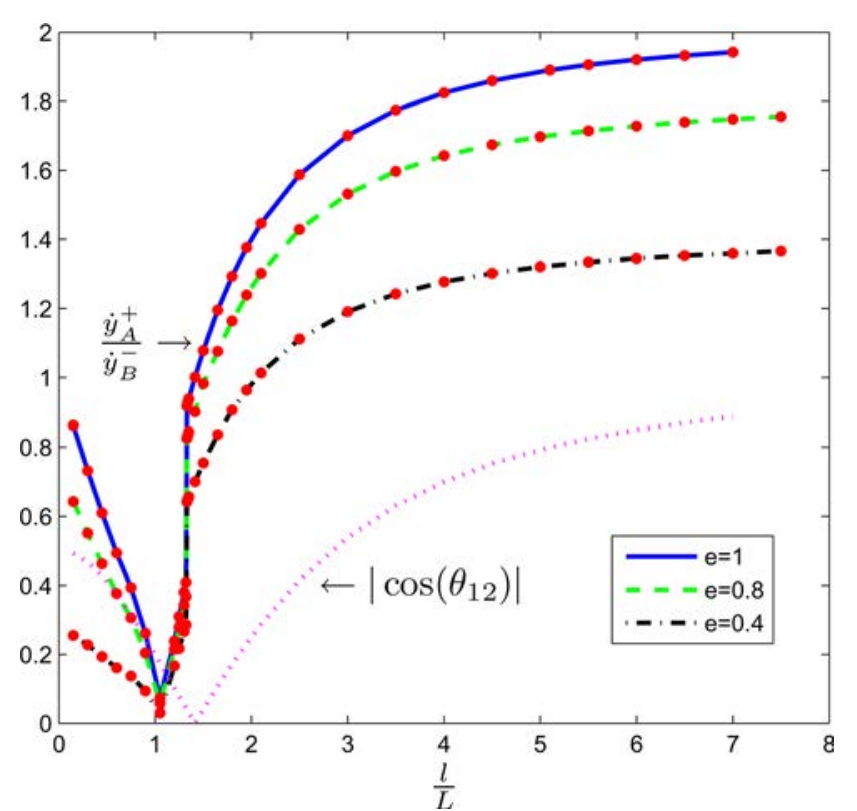

Fig. 10 Dispersion $d$ with varying $e_{n}^{*}$

as flat blocks always possess some slip. The LZB model confirms, in passing, that the tangential behavior during the impact may be complex with reversed tangential velocity, as illustrated in Fig. 11(b) for $l / L=0.3$. Kinematic restitution models usually are unable to properly take this into account $[13,14,16,23]$.

(10) In Fig. 10 the dispersion factor $d$ is depicted as a function of $l / L$, with $\mu=0.3$ and $\mu_{s}=0.5$, for three different values of $e_{n}^{*}$. It is remarkable that the normal dissipation has no effect on the global shape of the curve and that the minimum is independent of $e_{n}^{*}$. This leads one to infer that only the aspect ratio and the tangential stick/slip phenomena may play a role in the free-rocking dynamics. Figures $11(a)$ and $11(b)$ depict the horizontal velocity of the impacting point $A$ for various aspect ratios, before and after the impact. One sees from Fig. 11(b) that there are three distinct regions in the $(l / L, d)$ curves for the tangential behavior during the impact (the Darboux-Keller approach allows one to compute the velocities evolution

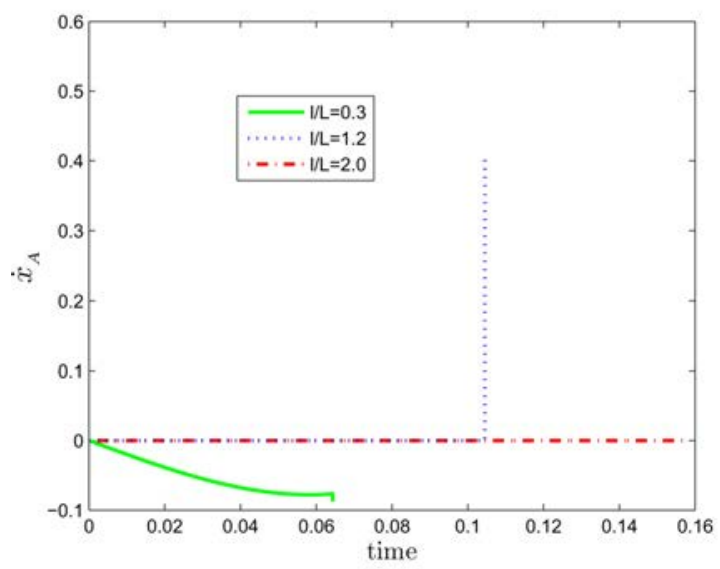

(a) Velocities before and after the impact during the impact, where the time scale is the contact forces impulse (in case of a multiple impact this is the impulse at the so-called primary contact point, see Ref. [25] for details)):

(a) slip with tangential velocity reversal for flat blocks $(l / L=0.3)$

(b) slip with unidirectional tangential velocity for moderately slender blocks $(l / L=1.2)$

(c) stick for slender blocks $(l / L=2)$

(11) In Figs. 12(a)-12(c) are reported the values of $d$ computed from the fitted parameters found in Sec. 6 in Ref. [35], where experimental results from Refs. $[3,4,44]$ are used to validate the impact model of Sec. 4 . All the details about the experimental data and the comparisons can be found in Ref. [35]. The experiments concern free-rocking of blue granite [4], steel [3], and concrete [44] block/ground systems. The fitted parameters are used to compute $d$ from the simulation. Since there is a good matching with the experimental results, one may consider that the computed values of $d$ match with the experimental ones, though the experimental values of pre- and post-impact velocities are not directly available from the measurements reported in Refs. [3,4,44]. Unfortunately little experimental data for slender blocks are available in the literature. No results for flat blocks have been published yet to the best of our knowledge. The results plotted in Figs. 12(a)-12(c) prove that the numerical calculations provide a very good prediction of the experimental works. We do not present other experimental validations because this would bring us outside the scope of this paper. As mentioned above, there have already been several detailed such validations published in Refs. [26-29,36] for various chains of balls and the bouncing dimer, and in the report [35] for the rocking block.

Remark 3. Reference [34] and Refs. [27,36] deal with the dynamics of a dimer that bounces on a vibrating table, with friction. In both papers, it has been shown that the dimer motion also possesses a critical aspect ratio that determines two different types of dynamical behaviors (see Fig. 11 in Ref. [27] and Fig. 4(a) in Ref. [34]). Though the kinetic angle is not used in these papers and despite the fact that the dimer and the block are different systems (see Remark 1), these aspect ratios correspond to some critical kinetic angle values of the dimer constraints. The results in this section show that the

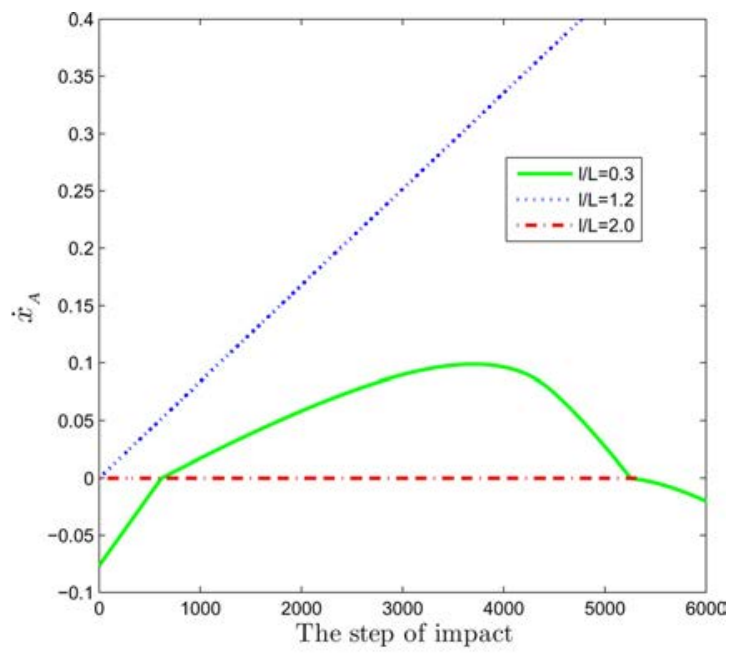

(b) Velocities during the impact

Fig. 11 Horizontal velocity at $A, \mu=0.3, \mu_{s}=0.5, e_{n}^{*}=1$ 


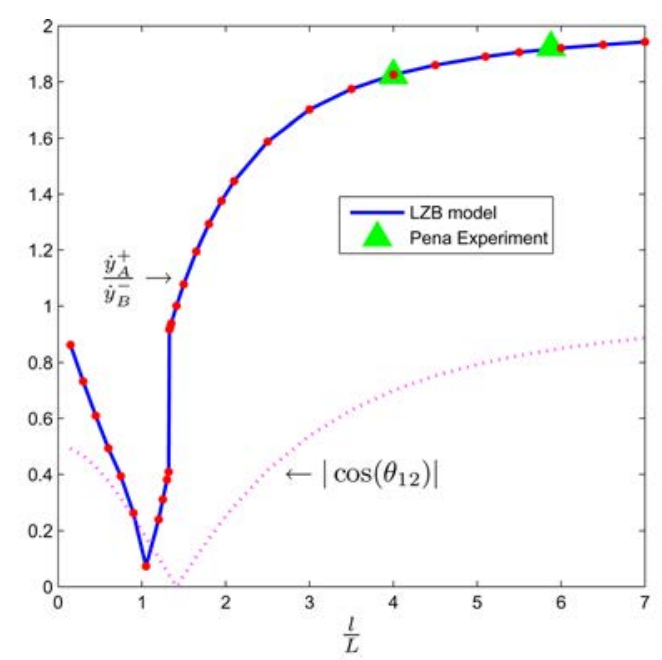

(a) From the experimental data in [4] (fitted parameters)

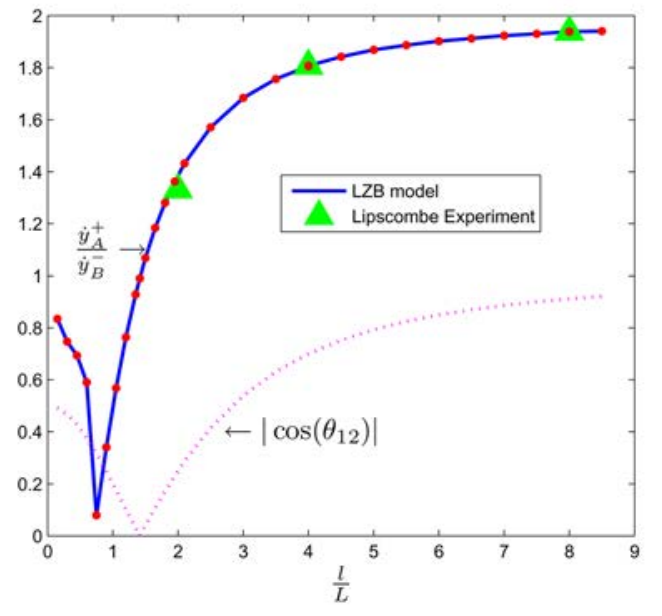

(b) From the experimental data in [3] (fitted parameters)

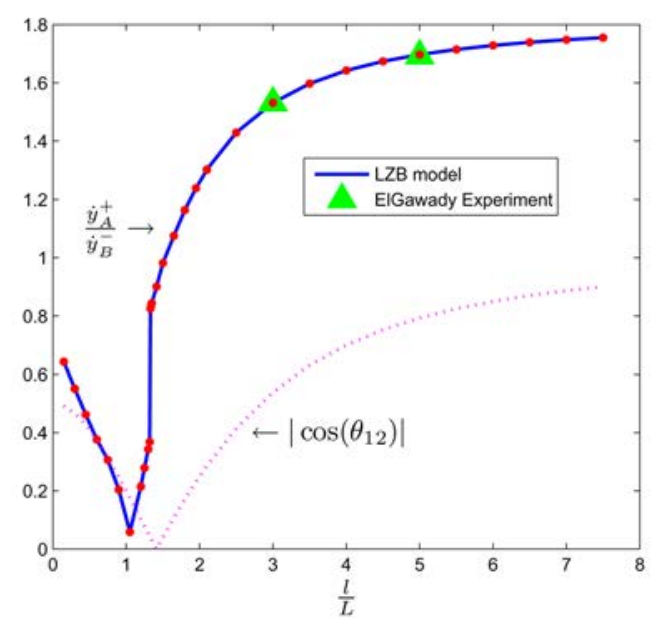

(c) From the experimental data in [44] (fitted parameters)

Fig. 12 Dispersion $d$ and experimental data

free-rocking motion of a planar block also exhibits some critical kinetic angles.

\section{Conclusions}

This paper deals with the free-rocking dynamics of planar blocks, which constitute an interesting example of a multiple impact with friction. The objective of this paper is twofold: first to provide new insights on the multiple impact phenomenon with Coulomb friction, and second to better understand the rocking block dynamics that are widely used in the Earthquake Engineering literature to study the behavior of structures subjected to earthquakes. The numerical results of this paper (see also the research report, Ref. [35]) prove that the multiple impact model introduced in Refs. [25-28,36] is able to reproduce a rich set of dynamical behaviors, with a limited set of parameters per contact. These simulation results also prove that the kinetic angle between the two constraint surfaces at a two-impact is a fundamental parameter, which allows one to characterize the energy dispersion at the contact/impact points. A critical value of the kinetic angle (evolving from $\theta_{c r}=\pi / 2 \mathrm{rad}$ in the frictionless case to $\theta_{c r}=\pi / 2.5 \mathrm{rad}$ for high enough friction) is exhibited. This value allows one to split the planar block into two classes: flat and slender blocks. Despite the role of the kinetic angle for frictionless constraints has been identified since a long time in the literature on multiple impact (in relation with the property of continuous dependence of trajectories on initial conditions), its role when multivalued Coulomb friction is considered has not been studied. This work (and the study on the dimer dynamics in Ref. [27]) proves that even in the presence of friction, some critical kinetic angle values (equivalently for the planar block: some critical aspect ratios) do exist. Thorough comparisons between numerical and experimental data have been done elsewhere [35] and prove that the multiple impact model and the numerical code that we use provide quite good predictions for free-rocking and for the onset of rocking (moving ground with harmonic excitation). Future work should be dedicated to extend this study to the three-dimensional block, which involves much more complex dynamics [45] and, therefore, deserves specific developments and analysis.

\section{Acknowledgment}

This work was performed with the support of the China/France NSFC/ANR project Multiple Impact, ANR-08-BLAN-0321-01. Hongjian Zhang was funded by the China Scholarship Council No. 2009601276 .

\section{References}

[1] Andreaus, U., and Casini, P., 1999, "On the Rocking-Uplifting Motion of a Rigid Block in Free and Forced Motion: Influence of Sliding and Bouncing," Acta. Mech., 138, pp. 219-241. 
[2] Housner, G. W., 1963, "The Behaviour of Inverted Pendulum Structures During Earthquakes,” Bull. Seismol. Soc. Am., 53(2), pp. 403-417.

[3] Lipscombe, P. R., and Pellegrino, S., 1993, "Free Rocking of Prismatic Blocks," J. Eng. Mech., 119(7), pp. 1387-1410.

[4] Pena, F., Lourenco, P. B., and Campos-Costa, A., 2008, "Experimenta Dynamic Behavior of Free-Standing Multi-Block Structures Under Seismic Loadings," J. Earthquake Eng., 12, pp. 953-979.

[5] Prieto, F., and Lourenco, P. B., 2005, "On the Rocking Behavior of Rigid Objects," Meccanica, 40, pp. 121-133.

[6] Shi, B., Anooshenhpoor, A., Zeng, Y., and Brune, J. N., 1996, "Rocking and Overturning of Precariously Balanced Rocks by Earthquakes," Bull. Seismol. Soc. Am., 86(5), pp. 1364-1371.

[7] Tso, W. K., and Wong, C. M., 1989, "Steady State Rocking Response of Rigid Blocks. Part 1: Analysis," Earthquake Eng. Struct. Dyn., 18(1), pp. 89-106.

[8] Tso, W. K., and Wong, C. M., 1989, "Steady State Rocking Response of Rigid Blocks. Part 2: Experiment," Earthquake Eng Struct. Dyn. 18(1), pp. 107-120.

[9] Yilmaz, C., Gharib, M., and Hurmuzlu, Y., 2009, "Solving Frictionless Rocking Block Problem With Multiple Impacts," Proc. R. Soc. London, Ser. A, 465, pp. 3323-3339.

[10] Yim, C. S., Chopra, A. K., and Penzien, J., 1980, "Rocking Response of Rigid Blocks to Earthquakes," Earthquake Eng. Struct. Dyn., 8(6), pp. 565-587.

[11] Heidenreich, B., 2004, "Small and Half-Scale Experimental Studies of Rockfall Impacts on Sandy Slopes," Ph.D. thesis, Ecole Polytechnique Fédérale de Lausanne, Lausanne, Switzerland.

[12] Brach, R. M., 1991, Mechanical Impact Dynamics, John Wiley, New-York.

[13] Brogliato, B., 1999, Nonsmooth Mechanics, 2nd ed., Springer, London.

[14] Brogliato, B., Zhang, H., and Liu, C., 2012, "Analysis of a Generalized Kinematic Impact Law for Multibody-Multicontact Systems, With Application to the Planar Rocking Block and Chains of Balls," Multibody Syst. Dyn., 27, pp. 351-382.

[15] Chatterjee, A., and Ruina, A., 1998, "A New Algebraic Rigid-Body Collision Law Based on Impulse Space Considerations,” ASME J. Appl. Mech., 65, pp. 939-951.

[16] Djerassi, S., 2009, "Collision With Friction; Part A: Newton's Hypothesis," Multibody Syst. Dyn., 29, pp. 37-54.

[17] Glocker, C., 2004, "Concepts for Modeling Impacts Without Friction," Acta Mech., 168, pp. 1-19.

[18] Modarres Najafabadi, S. A., Kövecses, J., and Angeles, J., 2008, "Generalization of the Energetic Coefficient of Restitution for Contacts in Multibody Systems," ASME J. Comput. Nonlinear Dyn., 3, p. 041008

[19] Modarres Najafabadi, S. A., Kövecses, J., and Angeles, J., 2008, "Impacts in Multibody Systems: Modeling and Experiments," Multibody Syst. Dyn., 20, pp. $163-176$.

[20] Modarres Najafabadi, S. A., Kövecses, J., and Angeles, J., 2007, "Energy Analysis and Decoupling in Three-Dimensional Impacts of Multibody Systems," ASME J. Appl. Mech., 74, pp. 845-851.

[21] Lubarda, V., 2010, "The Bounds on the Coefficients of Restitution for the Frictional Impact of Rigid Pendulum Against a Fixed Surface," ASME J. Appl Mech., 77(1), pp. 1-7.

[22] Payr, M., and Glocker, C., 2005, "Experimental Treatment of Multiple Contact Collisions," Proceedings of the Euromech Conference ENOC, Eindhoven, August 7-12, pp. 450-459.

[23] Stronge, W. J., 2000, Impact Mechanics, Cambridge University Press, Cambridge, UK
[24] Leine, R., and van de Wouw, N., 2008, "Stability Properties of Equilibrium Sets of Non-Linear Mechanical Systems With Dry Friction and Impact," Nonlinear Dyn., 51, pp. 551-583.

[25] Liu, C., Zhao, Z., and Brogliato, B., 2008, "Frictionless Multiple Impacts in Multibody Systems: Part I. Theoretical Framework,” Proc. R. Soc. London, Ser. A, 464(2100), pp. 3193-3211.

[26] Liu, C., Zhao, Z., and Brogliato, B., 2008, "Energy Dissipation and Dispersion Effects in a Granular Media," Phys. Rev. E, 78(3), p. 031307.

[27] Zhao, Z., Liu, C., and Brogliato, B., 2009, "Planar Dynamics of a Rigid Body System With Frictional Impacts. II. Qualitative Analysis and Numerical Simulations," Proc. R. Soc. London, Ser. A, 465(2107), pp. 2267-2292.

[28] Liu, C., Zhao, Z., and Brogliato, B., 2009, "Frictionless Multiple Impacts in Multibody Systems: Part II. Numerical Algorithm and Simulation Results," Proc. R. Soc. London, Ser. A, 465(2101), pp. 1-23.

[29] Nguyen, N. S., and Brogliato, B., 2012, "Shock Dynamics in Granular Chains: Numerical Simulations and Comparisons With Experimental Tests," Granular Matter, 14(3), pp. 341-362.

[30] Falcon, E., Laroche, C., Fauve, S., and Coste, S., 1998, "Collision of a 1-D Column of Beads With a Wall," Eur. Phys. J. B, 5(1), pp. 111-131.

[31] Nakagawa, M., Agui, J. H., Wu, D. T., and Extramiana, D. V., 2003, "Impulse Dispersion in a Tapered Granular Chain," Granular Matter, 4, pp. 167-174.

[32] Santibanez, F., Munoz, R., Caussarieu, A., Job, S., and Melo, F., 2011, "Experimental Evidence of Solitary Wave Interaction in Hertzian Chains," Phys. Rev. E, 84, p. 026604.

[33] Ceanga, V., and Hurmuzlu, Y., 2001, "A New Look at an Old Problem: Newton's Cradle,” ASME J. Appl. Mech., 68(4), pp. 575-583.

[34] Dorbolo, S., Volfson, D., Tsimring, L., and Kudrolli, A., 2005, "Dynamics of a Bouncing Dimer," Phys. Rev. Lett., 95(4), pp. 1-4.

[35] Zhang, H., and Brogliato, B., 2011, "The Planar Rocking Block: Analysis of Kinematic Restitution Laws, and a New Rigid-Body Impact Model With Friction," INRIA Research Report No. RR-7580.

[36] Liu, C., Zhao, Z., and Brogliato, B., 2008, "Variable Structure Dynamics in a Bouncing Dimer," INRIA Research Report No. 6718.

[37] Acary, V., and Brogliato, B., 2008, Numerical Simulation for Nonsmooth Dynamical Systems (Lecture Notes in Applied and Computational Mechanics), Vol. 35, Springer Verlag, Heidelberg, Germany.

[38] Heemels, W. P. M. H., Schumacher, J. M., and Weiland, S., 2000, "Linear Complementarity Systems," SIAM J. Appl. Math., 60, pp. 1234-1269.

[39] Paoli, L., 2005, "Continuous Dependence on Data for Vibro-Impact Problems," Math. Models Meth. Appl. Sci., 15(1), pp. 1-41.

[40] Stronge, W. J., 2000, "Chain Reaction From Impact on Coaxial Multibody Systems," ASME J. Appl. Mech., 67, pp. 632-635.

[41] "Siconos: A Software for Modeling and Simulation of Nonsmooth Dynamical Systems," 2012, http://siconos.gforge.inria.fr/

[42] Aslam, M., Godden, W. G., and Scalise, D. T., 1980, "Earthquake Rocking Response of Rigid Bodies,” ASCE J. Struct. Div., 106(2), pp. 377-392.

[43] Priestley, M. J. N., Evenson, R. J., and Carr, A. J., 1978, "Seismic Response Analysis of Structures Free to Rock on Their Foundations," Bull. New Zealand Seismol. Soc. Earthquake Eng., 11(3), pp. 141-150.

[44] ElGawady, M. A., Ma, Q., Butterworth, J. W., and Ingham, J., 2011, "Effects of Interface Material on the Performance of Free Rocking Blocks," Earthquake Eng. Struct. Dyn., 40(4), pp. 375-392.

[45] Chatzis, M. N., and Smyth, A. W., 2012, "Modeling of the 3D Rocking Problem,” Int. J. Non-Linear Mech., 47(4), pp. 85-98. 